\title{
A $p+1$ Method of Factoring
}

\author{
By H. C. Williams
}

\begin{abstract}
Let $N$ have a prime divisor $p$ such that $p+1$ has only small prime divisors. A method is described which will allow for the determination of $p$, given $N$. This method is analogous to the $p-1$ method of factoring which was described in 1974 by Pollard. The results of testing this method on a large number of composite numbers are also presented
\end{abstract}

1. Introduction. In 1974 Pollard [8] introduced a method of factorization which has since been called the $p-1$ factorization technique. Actually, the test was known to D. N. and D. H. Lehmer many years before this but it was never published because, without a fast computer, it was not possible to determine how effective it would be in practice. For the convenience of the reader we give a brief description of this test.

Suppose $N$ is a number to be factored and that $N$ has a prime factor $p$ such that

$$
p=\left(\prod_{i=1}^{k} q_{i}^{\alpha_{i}}\right)+1,
$$

where $q_{i}$ is the $i$ th prime and $q_{i}^{\alpha_{i}} \leqslant B_{1}$. Let $q_{i}^{\beta_{i}}$ be that power of $q_{i}$ such that $q_{i}^{\beta_{i}} \leqslant B_{1}$ and $q_{i}^{\beta_{i}+1}>B_{1}$ and put

$$
R=\prod_{i=1}^{k} q_{i}^{\beta_{i}} .
$$

Clearly, $p-1 \mid R$ and since $a^{p-1} \equiv 1(\bmod p)$ when $(N, a)=1$, we have $a^{R} \equiv 1$ $(\bmod p)$. Thus, $p \mid\left(N, a^{R}-1\right)$.

The algorithm now proceeds as follows. For a given $B_{1}$ put

$$
R=r_{1} r_{2} r_{3} \cdots r_{m}
$$

(for example, $\left.m=k, r_{i}=q_{i}^{\alpha_{i}}\right), a_{0}=a$, where $(a, N)=1$ and define

$$
a_{i} \equiv a_{i-1}^{r_{i}}(\bmod N)(i=1,2,3, \ldots, m) \text {. }
$$

The values of $a_{i}$ can be easily calculated by a power algorithm such as those mentioned in Knuth [4, p. 441ff.]. We now evaluate $a_{m} \equiv a^{R}(\bmod N)$ and $\left(a_{m}-1, N\right)$. Even for fairly small values of $B_{1}$ it frequently occurs that $\left(a_{m}-1, N\right)$ yields a nontrivial factor of $N$.

Pollard also gives in [8] two versions of a second step which can be appended to the above algorithm. We give one of these here.

Suppose instead of (1.1) we have

$$
p=s\left(\prod_{i=1}^{k} q_{i}^{\boldsymbol{\alpha}_{i}}\right)+1,
$$

Received March 30, 1981; revised September 28, 1981.

1980 Mathematics Subject Classification. Primary 10A25. 
where $s$ is a prime and $B_{1}<s \leqslant B_{2}$. In this case we have $p \mid\left(a_{m}^{s}-1, N\right)$. Let $\left\{s_{j}\right.$ : $j=1,2, \ldots, k\}$ be the ordered set of all primes such that $B_{1}<s_{j} \leqslant B_{2}$, and put $2 d_{j}=s_{j+1}-s_{j}$. Since the differences between successive primes increase very slowly, we see that there will not be very many distinct values for the $d_{j}$ 's. In fact, if we let $d(x)$ be the largest value of $d_{j}$ for all primes between 1 and $x$, we have $d(200000)=$ $43, d\left(10^{6}\right)=57$, and $d\left(4.444 \times 10^{12}\right)=326$; see Brent [1]. Thus, it is not too difficult to tabulate $a_{m}^{2 d_{j}}$ for all the distinct $d_{j}$. In fact, it is not really necessary to tabulate these values for all $d_{j}<d(x)$. The larger $d_{j}$ occur very seldom and the method is almost as fast if the table extends to only $K \log x$ for some moderate value of $K$ instead of $d(x)$ which seems to be $O\left((\log x)^{2}\right)$. This remark applies also to the second step of the $p+1$ method.

We calculate $b_{1} \equiv a_{m}^{s_{1}}(\bmod N)$ and define

$$
b_{j+1} \equiv a_{m}^{2 d} b_{j} \quad(\bmod N) .
$$

We now compute

$$
G_{t}=\left(\prod_{i=0}^{c}\left(b_{t+i}-1\right), N\right) \text { for } t=1, c+1,2 c+1, \ldots,\left[B_{2} / c\right] c+1 .
$$

Since $b_{j}=a_{m}^{s_{j}}(\bmod N)$, we see that $p$ must divide some $G_{i}$. Because greatest common divisors are more expensive to evaluate than products, we usually have $c>1$.

In [3] Guy and Conway suggest that, by using Lucas functions, the first step of the $p-1$ method can be converted into a factorization algorithm for finding a prime divisor $p$ of $N$ when $p+1$ has only small prime factors. In this paper we give a description of how this can be done. We also present a number of new factorizations which have been obtained by using either the $p-1$ or $p+1$ method. It should be mentioned here that John Brillhart and Earl Ecklund have also implemented a version of the first step of the $p+1$ method. However, in their few computer runs they were only able to find factors that had been previously discovered by the $p-1$ method. We also point out that a version of the method using the finite field of $p^{2}$ elements is also possible, if the reader wishes to avoid Lucas functions. Indeed, the author has been informed that R. P. Brent has an implementation of the $p+1$ method based on this interpretation.

2. The Lucas Functions. In order to develop the method and formulas required in the next section, we give here a description of some of the basic properties of the Lucas functions.

Let $P, Q$ be integers, and let $\alpha, \beta$ be the zeros of $x^{2}-P x+Q$. We define the Lucas functions by

$$
U_{n}(P, Q)=\left(\alpha^{n}-\beta^{n}\right) /(\alpha-\beta), \quad V_{n}(P, Q)=\alpha^{n}+\beta^{n} .
$$

We also put $\Delta=(\alpha-\beta)^{2}=P^{2}-4 Q$. When there is no doubt as to the values of the arguments $P$ and $Q$, we often omit them. These functions satisfy a large number of identities. We will require those given below

$$
\left\{\begin{array}{l}
U_{n+1}=P U_{n}-Q U_{n-1}, \\
V_{n+1}=P V_{n}-Q V_{n-1}
\end{array}\right.
$$




$$
\begin{aligned}
& \left\{\begin{array}{l}
U_{2 n}=V_{n} U_{n}, \\
V_{2 n}=V_{n}^{2}-2 Q^{n},
\end{array}\right. \\
& \left\{\begin{array}{l}
U_{2 n-1}=U_{n}^{2}-Q U_{n-1}^{2}, \\
V_{2 n-1}=V_{n} V_{n-1}-P Q^{n-1},
\end{array}\right. \\
& \left\{\begin{array}{l}
\Delta U_{n}=P V_{n}-2 Q V_{n-1}, \\
V_{n}=P U_{n}-2 Q U_{n-1},
\end{array}\right. \\
& \left\{\begin{array}{l}
U_{m+n}=U_{m} U_{n+1}-Q U_{m-1} U_{n}, \\
\Delta U_{m+n}=V_{m} V_{n+1}-Q V_{m-1} V_{n},
\end{array}\right. \\
& \left\{\begin{array}{l}
U_{n}\left(V_{k}(P, Q), Q^{k}\right)=U_{n k}(P, Q) / U_{k}(P, Q), \\
V_{n}\left(V_{k}(P, Q), Q^{k}\right)=V_{n k}(P, Q) .
\end{array}\right.
\end{aligned}
$$

These identities can all be verified by direct substitution from (2.1), using the simple facts that $P=\alpha+\beta$, and $Q=\alpha \beta$.

We also note that if $(N, Q)=1$ and $P^{\prime} Q \equiv P^{2}-2 Q(\bmod N)$, then $P^{\prime} \equiv \alpha / \beta+$ $\beta / \alpha$ and $Q^{\prime} \equiv \alpha / \beta \cdot \beta / \alpha=1$; hence,

$$
U_{2 m}(P, Q) \equiv P Q^{m-1} U_{m}\left(P^{\prime}, 1\right) \quad(\bmod N) .
$$

Finally, we need the following

TheOREM (SEe LeHMER [5]). If $p$ is an odd prime, $p \nmid Q$ and the Legendre symbol $(\Delta / p)=\varepsilon$, then

$$
\begin{aligned}
& U_{(p-\varepsilon) m}(P, Q) \equiv 0 \quad(\bmod p) \\
& V_{(p-\varepsilon) m}(P, Q) \equiv 2 Q^{m(1-\varepsilon) / 2} \quad(\bmod p) .
\end{aligned}
$$

3. The First Step of the Algorithm. Suppose that $p$ is a prime divisor of $N$ and

$$
p=\left(\prod_{i=1}^{k} q_{i}^{\alpha_{i}}\right)-1,
$$

where $q_{i}$ is again the $i$ th prime and $q_{i}^{\alpha_{i}} \leqslant B_{1}$. If $R$ is defined as in (1.2), we have $p+1 \mid R$. By the theorem of Section 2 we see that if $(Q, N)=1$ and $(\Delta / p)=-1$, then $p \mid U_{R}(P, Q)$, and therefore $p \mid\left(U_{R}(P, Q), N\right)$.

To find $U_{R}(P, Q)$, Guy and Conway seem to suggest that the first formulas of (2.2), (2.3), and (2.4) be used together with the second formula of (2.5) to obtain

$$
\begin{aligned}
U_{2 n-1} & =U_{n}^{2}-Q U_{n-1}^{2}, \\
U_{2 n} & =U_{n}\left(P U_{n}-2 Q U_{n-1}\right), \\
U_{2 n+1} & =P U_{2 n}-Q U_{2 n-1} .
\end{aligned}
$$

These formulas can be used in a power algorithm routine similar to that suggested by Lehmer [6] to find $U_{R}(P, Q)$. The problem with this method is that $R$ can be very large (for example, when $B_{1}=10^{5}, R>10^{43410}$ ), and it is difficult to store its value in the computer. Also, if $B_{1}$ is increased to obtain a new $R$ value, say $R^{\prime}$, we would 
have to start all over again at $U_{1}(P, Q)$ and $U_{2}(P, Q)$ to find $U_{R^{\prime}}(P, Q)$ instead of continuing on from $U_{R}(P, Q)$. These problems can be overcome by using a different technique.

If $p \mid U_{R}(P, Q)$, then by (2.3) $p \mid U_{2 R}(P, Q)$; thus, from (2.8) we have $p \mid U_{R}\left(P^{\prime}, 1\right)$. It follows that we lose no generality in assuming that $Q=1$. Further, by the Theorem of Section 2, we also have

$$
V_{(p-\varepsilon) m}(P, 1) \equiv 2 \quad(\bmod p)
$$

hence, if $p \mid U_{R}(P, 1)$, then $p \mid\left(V_{R}(P, 1)-2\right)$. We will assume throughout the remainder of this paper that $Q=1$ in our Lucas functions.

The first step of our $p+1$ algorithm is now the following:

Let $R=r_{1} r_{2} r_{3} \cdots r_{m}$ as above and find $P_{0}$ such that $\left(P_{0}^{2}-4, N\right)=1$. Define $V_{n}(P)=V_{n}(P, 1), U_{n}(P)=U_{n}(P, 1)$ and

$$
P_{j} \equiv V_{r_{j}}\left(P_{j-1}\right) \quad(\bmod N)(j=1,2,3, \ldots, m)
$$

By the second formula of (2.7), we see that

$$
P_{m} \equiv V_{R}\left(P_{0}\right) \quad(\bmod N) \text {. }
$$

We then calculate $\left(P_{m}-2, N\right)$.

To find $V_{r}=V_{r}(P)$ from $P$ we need only use the formulas

$$
\left\{\begin{array}{l}
V_{2 f-1} \equiv V_{f} V_{f-1}-P, \\
V_{2 f} \equiv V_{f}^{2}-2 \\
V_{2 f+1} \equiv P V_{f}^{2}-V_{f} V_{f-1}-P \quad(\bmod N),
\end{array}\right.
$$

(see the second formulas of (2.2), (2.3), and (2.4)).

Let

$$
r=\sum_{i=0}^{t} b_{i} 2^{t-i} \quad\left(b_{i}=0,1\right),
$$

$f_{0}=1$, and $f_{k+1}=2 f_{k}+b_{k+1}$; then $f_{t}=r$. Also, if $V_{0}(P)=2, V_{1}(P)=P$, then, to find the pair $\left(V_{f_{k+1}}, V_{f_{k+1}-1}\right)$ from $\left(V_{f_{k}}, V_{f_{k}-1}\right)$, we need only use the formula

$$
\left(V_{f_{k+1}}, V_{f_{k+1}-1}\right)= \begin{cases}\left(V_{2 f_{k}}, V_{2 f_{k}-1}\right) & \text { when } b_{k+1}=0, \\ \left(V_{2 f_{k}+1}, V_{2 f_{k}}\right) & \text { when } b_{k+1}=1,\end{cases}
$$

together with (3.2).

4. The Second Step of the Algorithm. Suppose

$$
p=s\left(\prod_{i=1}^{k} q_{i}^{\alpha_{i}}\right)-1
$$

where $s$ is a prime, and $B_{1}<s \leqslant B_{2}$. Define $s_{j}$ and $2 d_{j}$ as in Section 1. If $(\Delta / p)=-1$ and $p \nmid P_{m}-2$, then $p \mid\left(U_{s}\left(P_{m}\right), N\right)$ by (2.7) and (3.1).

Let $U[n] \equiv U_{n}\left(P_{m}\right), V[n] \equiv V_{n}\left(P_{m}\right)(\bmod N)$, and tabulate $U\left[2 d_{j}-1\right], U\left[2 d_{j}\right]$, $U\left[2 d_{j}+1\right]$ for the distinct $d_{j}$ by using

$$
U[0]=0, \quad U[1]=1 \text { and } U[n+1]=P_{m} U[n]-U[n-1] .
$$


Put

$$
T\left[s_{i}\right] \equiv \Delta U_{s_{i}}\left(P_{m}\right)=\Delta U_{s_{i} R}\left(P_{0}\right) / U_{R}\left(P_{0}\right) \quad(\bmod N),
$$

by the first formula of (2.7) and (3.1). From the second formula of (2.6), we have

$$
\left\{\begin{array}{l}
T\left[s_{1}\right] \equiv P_{m} V\left[s_{1}\right]-2 V\left[s_{1}-1\right] \\
T\left[s_{1}-1\right] \equiv 2 V\left[s_{1}\right]-P_{m} V\left[s_{1}-1\right](\bmod N)
\end{array}\right.
$$

and from the second formula of (2.6) we get

$$
\left\{\begin{array}{l}
T\left[s_{i+1}\right] \equiv T\left[s_{i}\right] U\left[2 d_{i}+1\right]-T\left[s_{i}-1\right] U\left[2 d_{i}\right] \\
T\left[s_{i+1}-1\right] \equiv T\left[s_{i}\right] U\left[2 d_{i}\right]-T\left[s_{i}-1\right] U\left[2 d_{i}-1\right](\bmod N)
\end{array}\right.
$$

Thus, to execute the second step of the algorithm we need only use (4.2) and (4.3) to obtain $T\left[s_{i}\right], i=1,2,3, \cdots$, and then evaluate

$$
H_{t}=\left(\prod_{i=0}^{c} T\left[s_{i+t}\right], N\right)
$$

for $t=1, c+1,2 c+1, \ldots, c\left[B_{2} / c\right]+1$. We must have $p \mid H_{i}$ for some $i$ if $p$ satisfies (4.1) and $(\Delta / p)=-1$.

5. Implementation and Results. One of the difficulties in implementing the $p+1$ algorithm of Sections 3 and 4 is the possibility that $p$ in (4.1) is such that $\left(P_{0}^{2}-4 / p\right)=+1$ for the selected value of $P_{0}$. There is no way of knowing beforehand that this will not occur. If we assume that the values of $P_{0}$ such that $(\Delta / p)=-1$ are randomly distributed, the probability that $(\Delta / p)=1$ is the same as the probability that $(\Delta / p)=-1$, i.e., $\frac{1}{2}$. Thus the probability that $\left(\Delta_{i} / p\right)=1$, $i=1,2,3, \ldots, n-1$, for each of $n$ trials at a $P_{0}$ value and $\left(\Delta_{n} / p\right)=-1$ for the $n$th trial is $\left(\frac{1}{2}\right)^{n}$. (We assume that the $P_{0}$ values selected are independent.) It follows that the probability that we will find some $\Delta_{i}$ such that $\left(\Delta_{i} / p\right)=-1$ after at most $n$ trials at a $P_{0}$ value is

$$
\sum_{i=1}^{n}\left(\frac{1}{2}\right)^{i}=1-\left(\frac{1}{2}\right)^{n}
$$

Thus, if $N$ has a prime factor $p$ which satisfies (4.1), and we use the algorithm of Section 3 with three trials at a $P_{0}$ value, we would expect to find that $p \mid\left(P_{m}-2, N\right)$ for seven of every eight such $N$ tested. The referee has pointed out that, instead of making three guesses at $P_{0}$, one could make many guesses to obtain $\Delta_{1}, \Delta_{2}, \Delta_{3}, \ldots$ as possible values of $\Delta$ for which $(\Delta / p)=-1$. One could then, by time sharing, test each of these $\Delta_{k}$ values a fraction $\beta_{k}$ of the time, where, of course, $\Sigma_{i=1}^{\infty} \beta_{i}=1$. Let $T_{0}$ (a function of the largest prime factor of $p+1$ ) be the time required by the algorithm if we were able to choose a $\Delta$ for which $(\Delta / p)=-1$. Then the time-sharing algorithm succeeds with probability 1 in an expected time

$$
T=T_{0} \sum_{k=1}^{\infty} \frac{1}{2^{k} \beta_{k}}
$$


We naturally wish to select the $\beta_{k}$ 's in such a way that $T$ is minimized. We note that by Cauchy's inequality

$$
\left(\sum_{k=1}^{\infty} \frac{1}{2^{k} \beta_{k}}\right)\left(\sum_{k=1}^{\infty} \beta_{k}\right) \geqslant\left(\sum_{k=1}^{\infty}\left(\frac{1}{2^{k} \beta_{k}}\right)^{1 / 2} \beta_{k}^{1 / 2}\right)^{2}
$$

hence,

$$
\sum_{k=1}^{\infty} \frac{1}{2^{k} \beta_{k}} \geqslant\left(\sum_{k=1}^{\infty} 2^{-k / 2}\right)^{2}=(\sqrt{2}+1)^{2} .
$$

Thus, an optimal choice of $\beta_{k}$ is $\beta_{k}=2^{-k / 2}(\sqrt{2}-1)$. Compared to taking 3 equally weighted trials, this method is slower when both succeed (ratio $3+2 \sqrt{2}: 3$ ) but it succeeds with probability 1 instead of $7 / 8$.

Both the $p-1$ and the $p+1$ methods were implemented on an AMDAHL 470-V7 computer and run with $c$ in (1.3) and (4.4) put equal to 14 and $B_{1}=10^{5}$, $B_{2}=2 \times 10^{5}$. Since the $p+1$ method is much slower (two times slower for Step 1 and about four times slower for Step 2) than the $p-1$ method, we always ran the $p-1$ method first on any given $N$ value.

Our programs were run on a total of 497 numbers. From an early version of a table of Brillhart et al. [2] (the most recent version includes the factors found here) we obtained 323 of these 497 numbers. These are factors of integers of the form $b^{n}-1$ with $b=2,3,5,7,10,11,12$. They were obtained by first dividing the main algebraic, including aurifeuillian, factor of $b^{n}-1$ by any of its algebraic divisors and then trial dividing by all primes up to $2^{35}$. Those remaining composite factors which were between 42 and 60 digits made up the 323 numbers referred to above. From an as yet unpublished table of factors of Fibonacci numbers, John Brillhart provided the author with the remaining 174 integers. Eighty-four of these are factors of the Fibonacci numbers $U_{n}(n=1,2,3, \ldots, 1000)$, where $U_{m+1}=U_{m}+U_{m-1}$ and $U_{0}=U_{1}=1$, and 90 are factors of the Lucas numbers $V_{n}(n=1,2,3, \ldots, 500)$, where $V_{m+1}=V_{m}+V_{m-1}$ and $V_{0}=2, V_{1}=1$. These numbers are between 41 and 80 digits in length and were known to have no divisors less than $2^{32}$ and no algebraic factors.

\begin{tabular}{|c|c|c|c|}
\hline$b$ & $m$ & $b, m L$ & $b, m M$ \\
\hline 2 & $4 k-2$ & $2^{2 k-1}-2^{k}+1$ & $2^{2 k-1}+2^{k}+1$ \\
\hline 3 & $6 k-3$ & $3^{2 k-1}-3^{k}+1$ & $3^{2 h-1}+3^{h}+1$ \\
\hline 5 & $10 k-5$ & $5^{4 k-2}-5^{3 k-1}+3 \cdot 5^{2 k-1}-5^{k}+1$ & $5^{4 h-2}+5^{3 h-1}+3 \cdot 5^{2 h-1}+5^{h}+1$ \\
\hline 6 & $12 k-6$ & $6^{4 k-2}-6^{3 k-1}+3 \cdot 6^{3 k-1}-6^{k}+1$ & $6^{4 h}{ }^{2}+6^{3 h-1}+3 \cdot 6^{3 h} 1+6^{h}+1$ \\
\hline 7 & $14 k-7$ & $\begin{array}{c}7^{6 k-3}-7^{5 k-2}+3 \cdot 7^{4 k-2}-7^{3 k-1} \\
+3 \cdot 7^{2 k-1}-7^{k}+1\end{array}$ & $\begin{aligned} 7^{6 h 3^{3}} & +7^{5 h-2}+3 \cdot 7^{4 h} 2+7^{3 h} 1 \\
& +3 \cdot 7^{2 h-1}+7^{h}+1\end{aligned}$ \\
\hline 10 & $20 k-10$ & $\begin{aligned} 10^{8 h-4} & -10^{7 k-3}+5 \cdot 10^{6 h-3} \\
& -2 \cdot 10^{5 k-2}+7 \cdot 10^{4 h-2} \\
& -2 \cdot 10^{3 k-1}+5 \cdot 10^{2 h} 1 \\
& -10^{h}+1\end{aligned}$ & $\begin{aligned} 10^{8 k-4} & +10^{7 h-3}+5 \cdot 10^{6 h-3} \\
& +2 \cdot 10^{5 k-2}+7 \cdot 10^{4 h}= \\
& +2 \cdot 10^{3 h} \cdot 1+5 \cdot 10^{2 h} 1 \\
& +10^{h}+1\end{aligned}$ \\
\hline 11 & $22 k-11$ & $\begin{aligned} 11^{10 h \cdots 5} & -11^{9 h-4}+5 \cdot 11^{8 h} 4 \\
& -11^{7 h-3}-11^{6 h \cdots 3}+11^{5 h} 2 \\
& -11^{4 h-2}-11^{3 h} 1+5 \cdot 11^{2 h} \quad \\
& -11^{h}+1\end{aligned}$ & $\begin{aligned} 11^{10 h} 5 & +11^{1 h \cdot 4}+5 \cdot 11^{\text {xh } 4} \\
& +11^{7 h}-11^{6 h} 3-11^{5 h} 2 \\
& -11^{4 h} 2+11^{3 h} 1+5 \cdot 11^{2 h} \quad \\
& +11^{h}+1\end{aligned}$ \\
\hline 12 & $6 k-3$ & $12^{2 h-1}-2^{2 h-1} 3^{h}+1$ & $12^{2 h} 1+2^{2 h} 3^{h}+1$ \\
\hline
\end{tabular}

TABLE 1 
The results of running the programs on these numbers are given in Tables 2,3, and 4. As is done in [2] we use the notation $b, m-$ and $b, m+$ to denote the numbers $b^{m}-1$ and $b^{m}+1$. The notation $b, m L$ and $b, m M$ for aurifeuillians is more complicated. We give their values (taken from [2]) in Table 1.

Note that $b, m L$ and $b, m M$ are factors of $b^{m}+1$ for $b=2,3,6,7,10,11,12$ and 5, $m L$ and 5, $m M$ are factors of $5^{m}-1$.

In the first column of Tables 2,3, and 4, we give the number which the composite integer $N$ divides. In the second column, we give the number of decimal digits in $N$. In the third and fourth columns, we give the prime factors of $N$ found by the computer program. A factor followed by an ' $E$ ' is one which was found by using Step 2 of the appropriate algorithm. An asterisk (*) in the first column is used to denote the fact that once the prime factors found in columns 3 and 4 had been divided into $N$, the remaining cofactor of $N$ is prime; hence, we have a complete factorization of the number in column 1. Primality of these numbers was established by using the program described in Williams and Judd [9]. Two asterisks (**) in the first column indicate that this cofactor of $N$, while composite, was subsequently factored by $M$. Wunderlich using the continued fraction method of Morrison and Brillhart [7]. It should be noted that the factors found here for 10,65 - and 10,69were found independently by G. J. Stevens in South Australia. He also used the $p-1$ method.

TABLE 2

\begin{tabular}{|c|c|c|c|c|}
\hline N divides & D & Factor (s) found by $p-1$ method & Factor (s) found by & $\mathrm{p}+1$ method \\
\hline 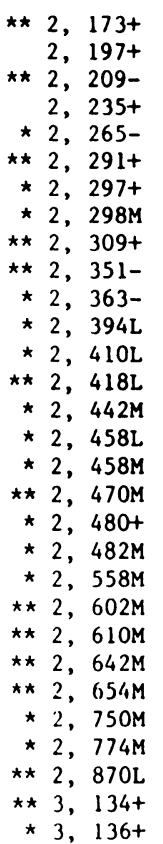 & $\begin{array}{l}46 \\
59 \\
54 \\
56 \\
52 \\
54 \\
44 \\
42 \\
44 \\
52 \\
56 \\
46 \\
49 \\
54 \\
49 \\
59 \\
50 \\
55 \\
58 \\
59 \\
54 \\
55 \\
55 \\
50 \\
54 \\
48 \\
59 \\
55 \\
50 \\
46\end{array}$ & $\begin{array}{l}47635010587 \\
197002597249 \\
94803416684681 \\
\\
197748738449921 \\
5636963037465601 \mathrm{E} \\
14641916303149 \mathrm{E} \\
2400744384937 \\
571890896913727 \\
75824014993 \\
152874915601 \\
61213422340181 \\
2291059412513 \\
84948746297, \quad 6211454306149 \\
44185520789894155033573 \mathrm{E} \\
87255998201 \\
137603804161 \\
76119208744309 \\
775844757937 \\
1621474400951381 \\
1193312900149 \\
168069194932501 \\
14512828061449 \\
4431960464101 \mathrm{E} \\
2670091735108484737\end{array}$ & $\begin{array}{l}236344687097 \\
87251820842149 \mathrm{E}\end{array}$ & · \\
\hline
\end{tabular}


TABLE 2 (continued)

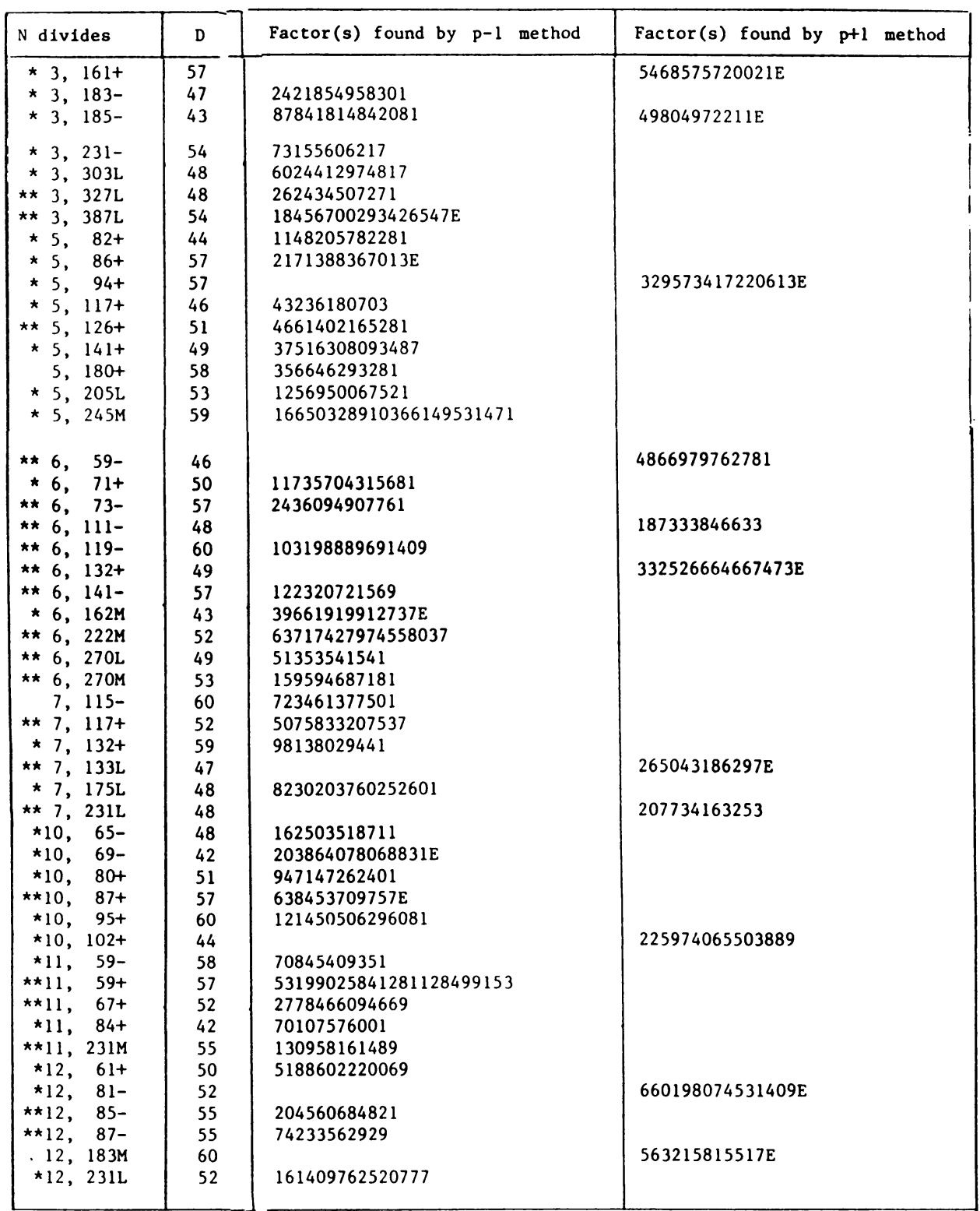

Factors were found for slightly over one quarter (134) of the 497 numbers tested. Most of these factors were found by the $p-1 \operatorname{method}(112$ vs. 32). This is what we would expect since (i) the $p-1$ method was used first and (ii) for the numbers in Table 2 there is a built-in bias toward the success of the $p-1$ test. This is because any prime divisor of $b^{m}-1$ which does not divide any algebraic factor of $b^{m}-1$ must be of the form $k m+1$. 


\section{TABLE 3}

\begin{tabular}{|c|c|c|c|}
\hline N & D & Factor (s) found by $p-1$ method & Factor (s) found by $p+1$ method \\
\hline${ }^{*} U_{247}$ & 46 & & 409100738617 \\
\hline${ }^{\star} \mathrm{U}_{307}$ & 55 & 5307027867738937 & \\
\hline${ }^{\star} \mathrm{U}_{313}$ & 59 & & 7901346123803597 \\
\hline$*_{323}$ & 52 & 85542646443577 & \\
\hline$\star_{343}$ & 57 & 5449038756620509 & \\
\hline$u_{361}$ & 72 & 6567762529,1196762644057 & \\
\hline$\star_{365}$ & 48 & 758275080626801 & \\
\hline$U_{367}$ & 59 & 5648966761 & 43397676601 \\
\hline $\mathrm{U}_{377}$ & 71 & 361575655741 & \\
\hline${ }^{\star} \mathrm{U}_{387}$ & 45 & & 14279673833 \\
\hline${ }^{\star} U_{403}$ & 65 & 42136290591640129 & \\
\hline $\mathrm{U}_{411}$ & 51 & $972663078773 \mathrm{E}$ & \\
\hline${ }^{\star U_{421}}$ & 75 & 45688564527041 & \\
\hline $\mathrm{v}_{455}$ & 55 & 36768087721 & \\
\hline $\mathrm{U}_{459}$ & 57 & 2043118036369 & \\
\hline${ }^{\star} U_{465}$ & 42 & $6936488411701, \quad 59666387254501$ & \\
\hline${ }^{\star} \mathrm{U}_{483}$ & 56 & & 1795220677069 \\
\hline $\mathrm{U}_{485}$ & 74 & $16892304192301, \quad 511715857773521$ & \\
\hline$*_{495}$ & 51 & & 1250839826281 \\
\hline $\mathrm{U}_{507}$ & 63 & 10069148777 & \\
\hline${ }^{\star} \mathrm{U}_{531}$ & 63 & & 2192843129417 \\
\hline${ }^{*} \mathrm{U}_{549}$ & 67 & $5883010433, \quad 80256319951861$ & 10424083697 \\
\hline$U_{555}$ & 53 & 49649320649221 & \\
\hline $\mathrm{U}_{567}$ & 68 & $49114912141, \quad 3936504300121$ & \\
\hline$U_{591}$ & 79 & 22221540969737 & \\
\hline $\mathrm{U}_{595}$ & 73 & $8310112721, \quad 9022425301$ & \\
\hline $\mathrm{U}_{633}$ & 73 & 41773163881 & \\
\hline${ }^{*} \mathrm{U}_{675}$ & 70 & 6641555895901 & \\
\hline $\mathrm{u}_{765}$ & 77 & 72208475461 & \\
\hline
\end{tabular}

The real problem with the $p+1$ test is the fact that it is quite slow. For our program we found that it was about nine times slower (when used three times for three different trials at $P_{0}$ value) than the $p-1$ test. Thus, one should probably use a higher bound for $B_{1}$ or $B_{2}$ for the $p-1$ test than for the $p+1$ test. We remark here, however, that if we had increased the $\max \left(B_{1}, B_{2}\right)$ to $10^{7}$, the $p-1$ test would very likely have found nine of the 32 factors found here by the $p+1$ test. This is because each of the remaining numbers $p$ is such that a prime which exceeds $10^{7}$ divides $p-1$. 
TABLE 4

\begin{tabular}{|c|c|c|c|}
\hline $\mathbf{N}$ & D & Factor (s) found by $p-1$ method & Factor (s) found by $p+1$ method \\
\hline$\star \mathrm{V}_{254}$ & 50 & & 347366417511089201 \\
\hline$\star v_{271}$ & 46 & 92206663291 & \\
\hline${ }^{\star} V_{283}$ & 56 & 252605941501 & \\
\hline$v_{299}$ & 46 & 143236388738249 & \\
\hline$v_{302}$ & 63 & 70963651961 & \\
\hline$v_{304}$ & 45 & 12441241017224321 & \\
\hline$v_{331}$ & 64 & 54184296181 & \\
\hline$v_{338}$ & 57 & & 404112157123 \\
\hline$\star V_{346}$ & 72 & $68520477202692467 \mathrm{E}$ & \\
\hline$\star v_{352}$ & 67 & 3891324187650256896001 & \\
\hline$v_{358}$ & 75 & 316590102769 & \\
\hline$v_{367}$ & 71 & & 19997474011 \\
\hline$\star V_{369}$ & 48 & 26024651929 & $18736753266019 E$ \\
\hline$v_{374}$ & 59 & 3827019260681 & \\
\hline$\star \mathrm{V}_{384}$ & 42 & & 1769526527 \\
\hline$\star V_{390}$ & 41 & 54975368761 & \\
\hline$v_{406}$ & 67 & 64690797641 & \\
\hline$\star V_{413}$ & 67 & 33637840386809 & \\
\hline$* V_{418}$ & 59 & 722601451307 & \\
\hline$v_{419}$ & 79 & 316722762859 & \\
\hline$v_{426}$ & 55 & & 1006118006507 \\
\hline$* V_{428}$ & 74 & 386610981607 & \\
\hline$* V_{460}$ & 69 & 28677143808961 & \\
\hline$\star V_{469}$ & 70 & 10812055185331 & \\
\hline$\star v_{477}$ & 66 & $49721203549 \mathrm{E}$ & 6430515046741 \\
\hline
\end{tabular}

6. Acknowledgements. The author wishes to thank John Brillhart and Sam Wagstaff for making available to him the tables referred to in Section 5.

Department of Computer Science

University of Manitoba

Winnipeg, Manitoba, Canada R3T 2N2

1. Richard P. BRent, “The first occurrence of certain large prime gaps," Math. Comp., v. 35, 1980, pp. 1435- 1436.

2. John Brillhart, D. H. Lehmer, J. L. Selfridge, Bryant Tuckerman \& S. S. Wagstaff, Jr., Factorizations of $b^{n} \pm 1, b=2,3,5,7,10,11,12$ Up to High Powers. (To appear.)

3. Richard K. GUY, "How to factor a number," Congressus Numerantium XVI, Proc. Fifth Manitoba Conf. on Numerical Math., Winnipeg, 1976, pp. 49-89.

4. Donald E. Knuth, The Art of Computer Programming, Vol. II, Seminumerical Algorithms, 2nd ed., Addison-Wesley, Reading, Mass., 1981.

5. D. H. Lehmer, “An extended theory of Lucas' functions," Ann. of Math. (2), v. 31, 1930, pp. 419-448.

6. D. H. Lehmer, "Computer technology applied to the theory of numbers," in Studies in Number Theory (W. J. LeVeque, ed.), Math. Assoc. Amer. Studies in Math., vol. 6, 1969, pp. 117-151.

7. Michael A. Morrison \& John Brillhart, "The factorization of $F_{7}$," Bull. Amer. Math. Soc., v. 77, 1971, p. 264.

8. J. M. Pollard, "Theorems on factorization and primality testing," Proc. Cambridge Philos. Soc., v. 76, 1974, pp. 521-528.

9. H. C. Williams \& J. S. JudD, "Some algorithms for prime testing using generalized Lehmer functions," Math. Comp., v. 30, 1976, pp. 867-886. 